\title{
Development of a collaborative optimization tool for the sizing design of aerospace structures
}

\author{
L. Guadagni ${ }^{1, a}$ \\ CNES, Rond-point de l'Espace, 91023 Evry, France
}

Received 30 October 2007, Accepted 15 February 2008

\begin{abstract}
The work introduces a computational method for the optimization of aerospace preliminary designs. It was developed considering a collaborative approach to solve the sizing optimization of a structure model defined by finite element method and by a large number of subsystems. A brief introduction explains how the collaborative optimization method can be used for general structural problem and how this approach can offer many advantages to the computation analyses. Central discussion is the description of an automatic procedure defined by three deterministic optimization codes and their interface systems with the MSC.Nastran environment. They decompose the structure complexity executing an interface algorithm for FE models searching an optimized solution for the structural subsystems respecting different kinds of constraints. Furthermore, in order to show the uses of the procedure in an industrial context, two realistic applications for the minimum design are also discussed. The results show a good comparison with the commercial solvers, taking out better results in less time processing and finding new alternative design configurations.
\end{abstract}

Key words: Collaborative optimization method; FE analysis; aerospace application

\section{Introduction}

Aerospace engineering is a complex system of different sciences working towards the definition of an aerospace design. Since the advent of aviation the structural problem was one of the more critical tasks for the engineers. In general a good structural solution was a good starting point from which all the other disciplines could adapt. The earliest studies, acting on the interaction between all the aerospace branches, show that a different approach is necessary. As the aerospace design is a multidisciplinary problem, the single disciplines are inserted in an iterative design process: any decision or choice made on a discipline has an effect on the other ones. Nowadays, this fact is well known in the engineering area and with the increase of the aerospace activities, more complex computational methods are been developed. Some good samples and solutions founded to solve complex phisical systems in the aerospace demain are available in different works $[1-3]$.

In a structural approach this means to take into account more detailed model in order to consider the phenomena inherent to all structural aspects; consequently the structural complexity of the design become an important aspect to face because of its influence on the solution method. In the aerospace industry, for example, looking

\footnotetext{
${ }^{a}$ Corresponding author: luca.guadagni@gmail.com
}

for a structural solution often means considering a large number of design requirements that can heavily modify the solution during the computation. In a mathematical context the design process turns out to be an optimization task definition; defining the structural design with a mathematical model, the target becomes to reach an optimal condition (i.e. a lighter structural configuration in a structural task) where the performances and the requirements have to be feasible and satisfied. There are a lot of programs available today and most of them are general purpose software based on finite element method (FEM) working with non-specific models. Sometimes the response of the optimization is too generic and a more specific solution is required.

In this article an optimization procedure for preliminary aerospace design is presented. The activity is focused on the sizing optimization of complex system defined by a large number of structural subsystems that are improved with dedicated external solvers using the finite element analysis (FEA) only for the static equilibrium determination. The process is iterative and in order to guarantee the effects of each subsystem analysis, the overall model is updated with the previous local results for each iteration. The procedure is a tool of external software working under the control of Multi Level Architectures (MLA) called Collaborative Optimization (CO). The last section of this work is dedicated to the analyses of complex aeronautical and space structures and to the results discussion. 


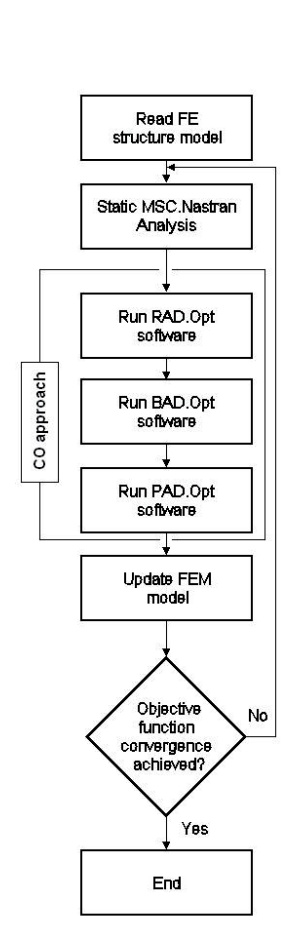

(a)

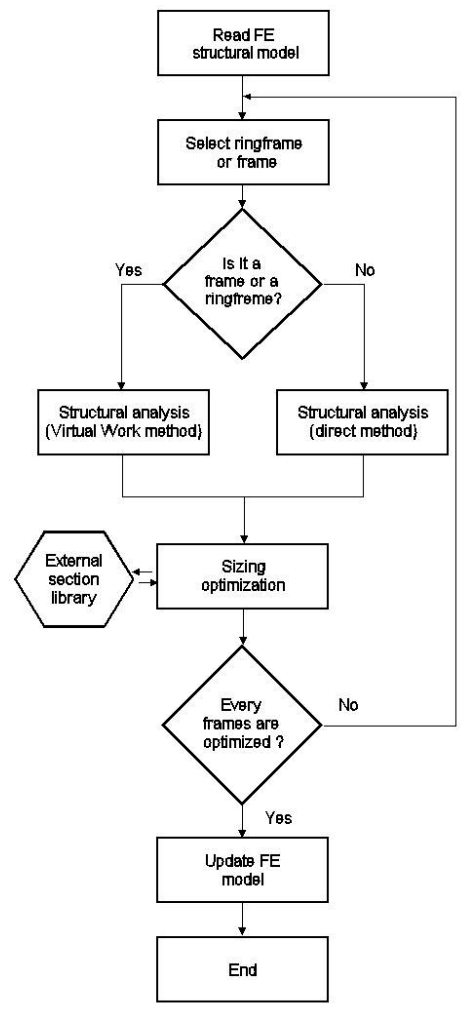

(b)

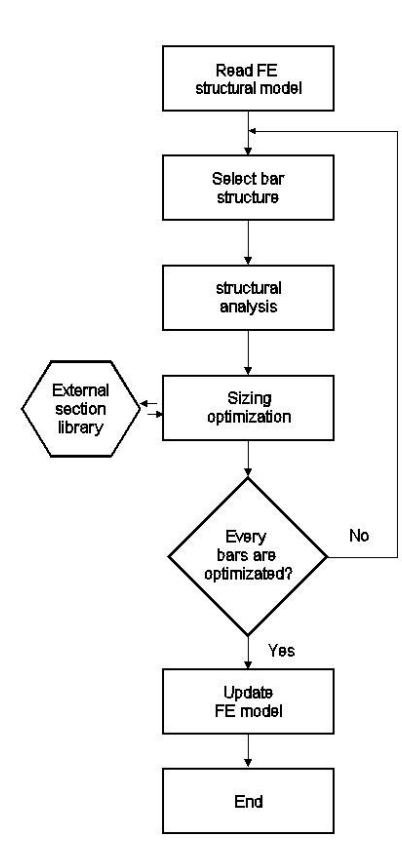

(c)

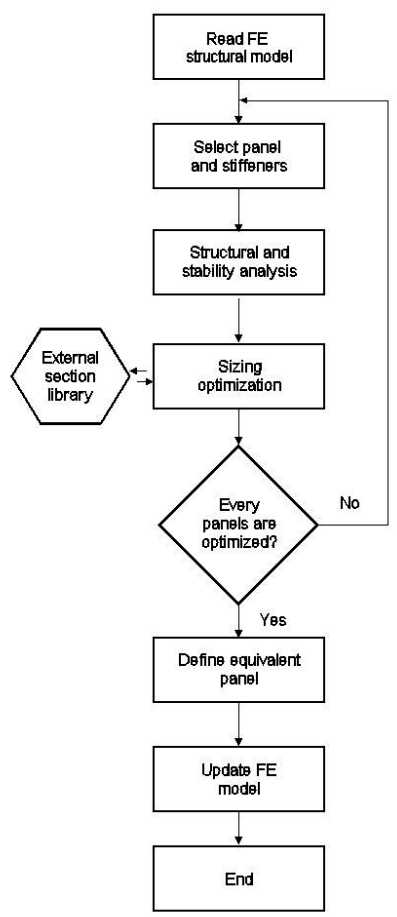

(d)

Fig. 1. The flow chart for the global procedure OPT.TOOL (a) and for the local optimization software: RAD.Opt (b), BAD.Opt (c) and PAD.Opt (d).

\section{The OPT.TOOL procedure}

In this article a Multidisciplinary Design Optimization (MDO) procedure with a $\mathrm{CO}$ architecture is used offering many advantages. The main idea is to disassemble the system to different structural subsystems and to consider these as single disciplines. In a CO context the theory imposes that the discipline solvers work as stand-alone, changing the whole process as well as a system of independent subsystem software [4]. The procedure developed here is an OPTimization TOOL (OPT.TOOL) dedicated to the aerospace applications. The software is not a general purpose optimizer, but a manager for local optimization solvers. The procedure was created to deal with sizing optimization tasks where: (1) the global structure is defined by a FEM with high level of complexity; (2) local solvers for optimal sizing are developed for specific subsystems; (3) the local optimal solutions are derived without using the FEA; (4) the optimization goal is the global weight minimization; (5) a large number of design variables and constraints are taken into account; (6) the FE model is updated with all optimal solutions from each external solvers. At the first step of its flow chart (Fig. 1a) a FEM properties reading is provided and in order to evaluate the stress conditions, a static equilibrium of the global model is performed using MSC.Nastran. Furthermore the execution of some external solvers in a $\mathrm{CO}$ environment are activated and the process ends with the optimal re- sults updating in a FEM file. Three optimization analyses for aerospace structures are available at the local level: (1) wing and fuselage frame (RAD.Opt); (2) beam and stringer elements (BAD.Opt); (3) stiffened and curved panels (PAD.Opt). All the external codes, the software manager and the algorithms were written by the author in C language using the Numerical Recipes Library in $C^{1}$.

\subsection{The external optimization software}

In this paragraph a description of the main common and specific properties for the external optimizators is reported. Each optimization program operates on the suitable elements only: after the property reading from the FE input file and the recovery of the stress conditions, a optimization solution is performed. At the end of the process the optimal results are updated into a new FEM. The flow charts are an assembly of modules defined by several complex algorithms interfacing with the FEM. The codes can distinguish between different type of FE elements (with one and two dimensions only) and automatically translates the mechanical properties for the solver. As the optimization considers the forces acting on the elements, the rest of the data are taken from the static FEA performed at the beginning. Afterwards the codes search

\footnotetext{
${ }^{1}$ Numerical Recipes Library in $C$ (2nd ed.) is a registered trade-mark by Cambridge University Press.
} 
an optimal configuration using the feasible direction algorithm (based on the gradient method) [5] respecting specific requirements. As a commercial software gives a single solution of the optimization problem, the codes are provided with an external library of cross section shapes. When needed it can be used to change the mechanical characteristics (i.e. for a stringer or stiffener element), besides having an improvement on the original model a set of alternative configurations is also available. The process ends with the updating of the optimal data into the global FEM properties. The first local software is a Ring frame Analysis and Design Optimizer (RAD.Opt), its target is to determie an optimal sizing of a frame with a specific shape without using the FEA computation, but resolving inherent closed formulation. As the structural analysis is tightly linked to the frame shape, and to give to the procedure more generality, two closed shapes are taken into account (Fig. 1b): (1) circular shape (ringframe) and (2) non-circular shape (frame). For both cases a specific structural analysis for the stress distribution determination is available and discussed in a previous work [6]. The second local solvers is the Beam Analysis and Design Optimizer (BAD.Opt) for the computation of structural optimization of longitudinal beam elements, like wing stringers or stiffened panel stiffeners (Fig. 1c). The last code is a Panel Analysis and Design Optimizer (PAD.Opt). The program's goal is the sizing optimization of stiffener panels in the case of a preliminary design (Fig. 1d). This means the software hasn't a complete theory for panel analysis, but it is only a way to predict an optimal configuration considering: (1) several geometrical (flat and curved panels, stiffened panels with a orthogonal grid) and load configurations; (2) different material characteristics (isotropic and composite material defined with the smeared theory); (3) failure modes for local skin, stiffener and global buckling (performed solving handbook formulas). Three groups of variables designs are considered for the optimum panel weight: (1) the skin thickness; (2) the stiffener cross sections; (3) the stiffener distance (between two subsequent stiffeners). For each program an optimization task on the specific structural components is performed. The algorithm can be defined by the following common system of equations:

$$
\begin{cases}W\left(\rho, d_{i}, l_{e}\right) \rightarrow W_{\min } & \text { objective function } \\ d_{i, \min } \leq d_{i} \leq d_{i, \max } & \text { geometric constraints } \\ \sigma_{e}(s) \leq \sigma_{a m m} & \text { stress constraints } \\ \sigma_{e}(s) \leq \sigma_{l, c r} & \text { local stability constraints } \\ \sigma_{e}(s) \leq \sigma_{g, c r} & \text { global stability constraints }\end{cases}
$$

where $\rho$ is the material density, $e$ the number of subsystem finite elements (located by the geometrical variable $s$ ) and $l_{e}$ the FE element lengths. The target of the optimization is to minimize the subsystem weight $W$ with respect to the design variables $d_{i}$ of the cross section and to geometrical $\left(d_{i, \min }\right.$ and $d_{i, \max }$ is the dimensional range by user's defined) and structural constraints, comparing these last ones with the ultimate yield stress of the materials $\left(\sigma_{a m m}\right)$. There is also a third group of constraints relative to the instability conditions. Each solver has some specific features to evaluate the local critical load $\sigma_{l, c r}$ on particular elements (i.e. the local instability of a panel stiffener) or the global one $\sigma_{g, c r}$ on the overall subsystems (i.e. the global instability of a stiffened panel). At the present time the software capabilities are limited to the structural analysis of metal alloy with isotropic or anisotropic properties.

\subsection{The collaborative approach}

OPT.TOOL is a multilevel software manager for the execution of the local optimizers working on specific substructures. The use of this method is based on the intuition to consider a problem classified as disciplinary with a multidisciplinary approach. This idea should appear in contradiction and counterproductive, but the CO can bring a lot of advantage to the structural optimization for complex FE systems. The three programs work on the same disciplines, but on different components. They appear to be independent, but as the components are together assembled and the global structure must be statically determined, a set of shared parameters are identifiable (i.e. all the forces and flows at the interface FE grids). These parameters influence the behaviour of the local optimizers acting on the local stress and instability constraints and realizing a little interdisciplinary data exchange. In relation to two different definitions of the interdisciplinary consistency constraints, two different $\mathrm{CO}$ methods $\left(\mathrm{CO}_{1}\right.$ and $\left.\mathrm{CO}_{2}\right)$ are developed by Alexandrov and Lewis $[7,8]$. In this work a $\mathrm{CO}_{1}$ approach is used (Fig. 2) bringing several benefits: (1) the local optimization always obtains a feasible design; (2) the interdisciplinary parameters are seen at the local level only (independent disciplines); (3) the interdisciplinary consistency constraints are defined with a linear function allowing the use a gradient based algorithm.

\section{OPT.TOOL analyses}

In this paragraph two OPT.TOOL analyses are discussed. As the software tool is developed to deal with realistic structural designs, the results are based on complex FE model and show the main OPT.TOOL features: (1) providing a multiconfiguration structural solution; (2) satisfying the requirements initially defined. The first problem refers to an aeronautical application, the second one to a space module and, in order to evaluate the procedure, a comparison analysis with a commercial optimizer is also presented on the last problem.

\subsection{Aeronautical wing structure}

The example presents a wing structure of a utility aircraft (Fig. 3a), loaded with a set of realistic loads: aerodynamic pressure, gravitational forces and inside system weights. The loads are performed defining the load factor $n$ in different flight condition as: normal $(n=+1)$, upside down 


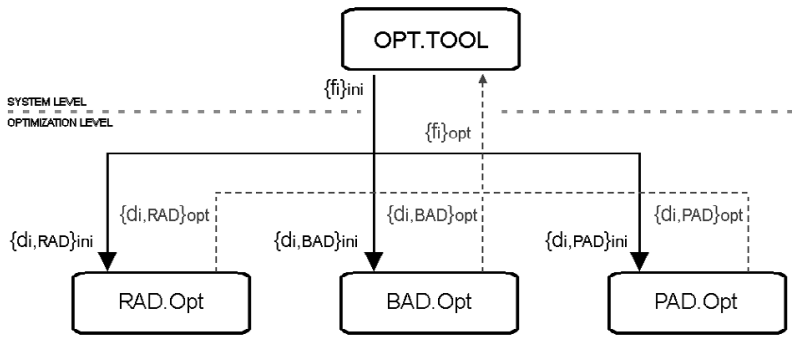

Fig. 2. The OPT.TOOL collaborative architecture. it reads the local design varables $\{f\}_{i n i}$ and takes out the optimal values $\{f\}_{\text {opt }}$.

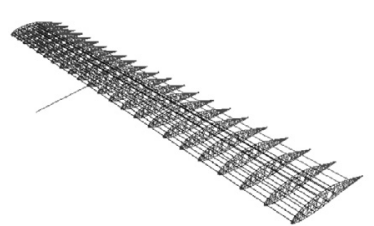

(a)

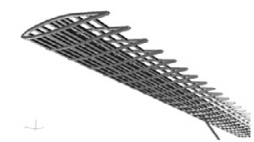

(c)

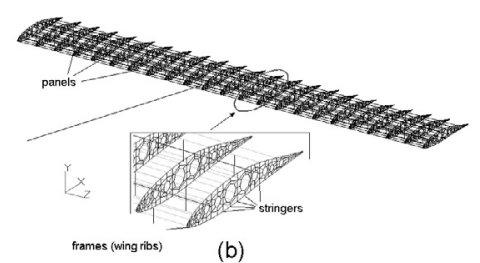

(b)

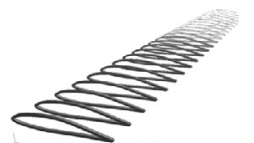

(d)
Fig. 3. Outline of an aircraft wing (a) with the details of three structural groups: the panels (b), the stringers (c) and the wing ribs $(d)$.

$(n=-1)$, hard landing $(n=+2.5)$ and over the maximum load factor $(n=+3.9)$. The following substructures are present in the model: 48 panels (Fig. 3b), 17 longitudinal stringers (Fig. 3c), 23 wing frames (Fig. 3d). For this last group only the cross section of the closed-shaped frame around the wing web is taken into account for the analysis because of the small value of the wing web thickness. No ring frames or stiffened panels are in the model. The material is an aluminium alloy for all groups. In relation to a simuilar application developed by Piperni [9] and Craig [10], the aim of these examples is to show the feature of the OPT.TOOL procedure to determine a optimal configuration in relation to the following requirements: (1) all the load cases are acting (improbable situation); (2) specific range of values defined on the cross section and panel thickness (respectively $9 \mathrm{~mm}^{2} \leq A_{i}\left(d_{i}\right) \leq 900 \mathrm{~mm}^{2}$ and $1 \mathrm{~mm} \leq t_{p} \leq 100 \mathrm{~mm}$ ); (3) ultimate yield stress imposed to $270 \mathrm{MPa}$; (4) different safety factors for the structural $\left(s_{1}\right)$ and stability constraints $\left(s_{2}\right)$ (respectively $\left|\sigma\left(d_{i}\right)\right| \leq \frac{\sigma_{m a t}}{s_{1}}$ for the stress constraint and $\sigma\left(d_{i}\right) \leq \frac{\sigma_{c r}}{s_{2}}$ for stability one). A total number of 288 design variables are available for the optimization. Three analysis cases are performed in relation to different security factors: $s_{1}=s_{2}=1.0($ case 1$), s_{1}=s_{2}=1.2($ case 2$)$ and $s_{1}=s_{2}=1.3$ (case 3 ), where the graphs in Figure 4 are drawing respect to the computational iterations nedeed for the objective function convergence. Because of the acting loads, the global weight increase for the three cases (Tab. 1), while a light increase is available for the "set 1", a reference analysis with single load $n=+1$ and security factors $s_{1}=s_{2}=1.2$. The result shows that, in
Table 1. OPT.TOOL wing analysis: results for multi-load and single (set 1) load cases.

\begin{tabular}{lcccc}
\hline & case 1 & case 2 & case 3 & set 1 \\
\hline Start [kg] & 81.21 & 81.21 & 81.21 & 81.21 \\
OPT.TOOL [kg] & 128.03 & 128.06 & 128.52 & 105.79 \\
time & $1^{h} 02^{m}$ & $1^{h} 05^{m}$ & $1^{h} 03^{m}$ & $0^{h} 25^{m}$ \\
\hline
\end{tabular}

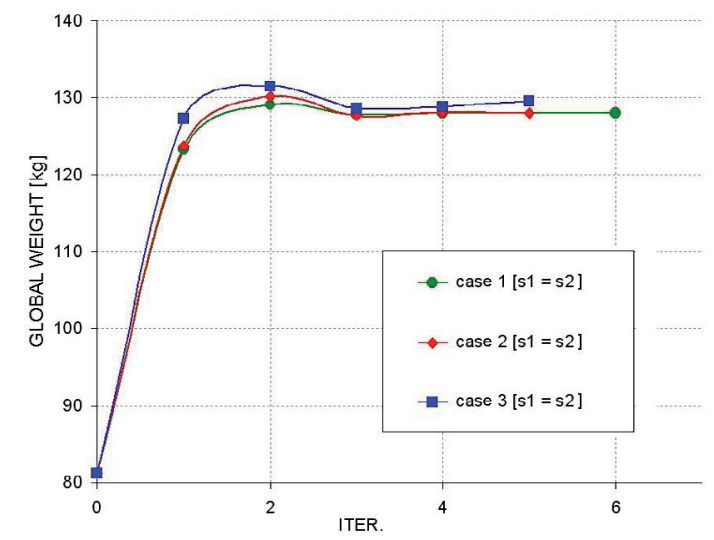

Fig. 4. OPT.TOOL analyses for a wing aircraft. Global weight graphs for different cases.

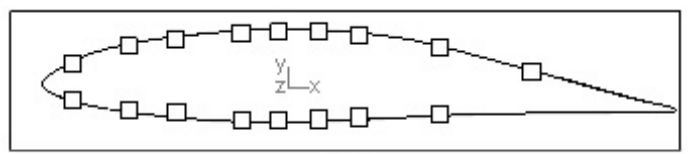

(a)

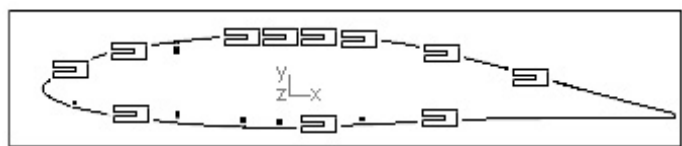

(b)

Fig. 5. OPT.TOOL wing analysis. Cross section (case 2) for initial (a) and optimal configuration (b).

order to consider a safe configuration and respecting all loads taken into account, a $20 \mathrm{~kg}$ of structure mass must be added. Another OPT.TOOL feature is the performing of a multiconfiguration solution. In this example the OPT .TOOL can generate different cross section typologies for the design variables of the wing section. From a homogeneous condition (Fig. 5a, rectangular cross sections) the solver goes towards a heterogeneous one (Fig. 5b rectangular and "C" cross section).

\subsection{Space module}

The example shows the OPT.TOOL computation performances for space applications. The structure is a space module of the International Space Station (ISS) designed by ALCATEL ALENIA Space. An example of the use of a CO approach to the aerospace application is available in Brown [11] (where a more generic optimization on 


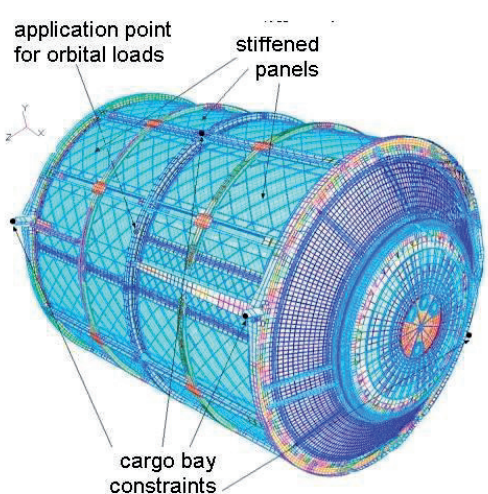

(a)

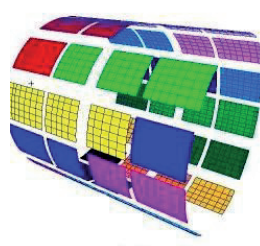

(b)

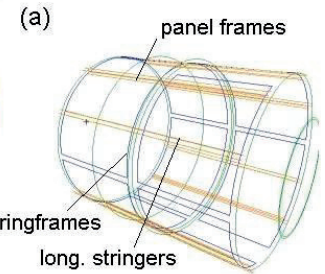

(c)
Fig. 6. Space structure model (a) with the panels (b) and the bar elements (c) details.

the global design of a space vehicle, with a comparison of three different methods, is reported) and in Wallace [12], where the philosophy of the CO design is applied as well as it was done for this work. The FE model ${ }^{2}$ has an high definition degree and was created taking into account (1) the primary structure (no inside structures are presented) and (2) a large set of loads (Fig. 6a). These are taken from a realistic situation of typical space mission: lift- off and cargo bay loads, internal pressure, berthing conditions and static connection forces to the Space Station. Two results are performed: (a) a sample computation of the optimal configuration and (b) a comparison with a commercial optimizer. The first analysis is based on the sizing optimization of the following three design structural groups: 44 biaxial blade stiffened panels (Fig. 6b), 32 longitudinal stringers, 13 ring frames and general shape frames (Fig. 6c). All the materials are aerospace isotropic alloy and composites. As reported before, for the wing example, also for this problem different requirements are imposed: (1) all the load cases are activated; (2) specific range defined on the design variables of the cross section of stringers, frames, panel stiffeners and panel thickness (respectively $8 \mathrm{~mm} \leq d_{s t}, d_{f r}, d_{p s}, t_{p} \leq 50 \mathrm{~mm}$ ); (3) ultimate yield stress imposed to $270 \mathrm{MPa}$; (4) structural and stability security factors fixed respectively to $s_{1}=s_{2}=1.0$. Overall the procedure has to solve an optimization problem with 666 variables designs. In Figure $8 \mathrm{a}$ two lines are reported to indicate the initial FE model weight (dotted line) and the actual one considering the mass of the all component features that, requiring a high FEM definition, they are described into the OPT.TOOL input file (i.e. the stiffened panels etc.). The results show a small increase of the global model

2 ALCATEL ALENIA Space is the owner of the FE model used for the analyses.

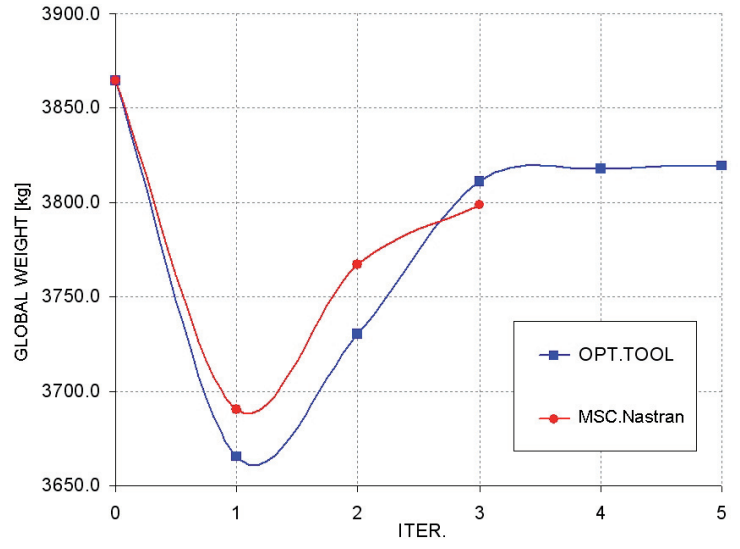

Fig. 7. Result comparison of the global weight on a space structure.

weight. The single code behaviours show (Fig. 8b) an increase of the panel group weight (dotted line), on the contrary a decrease for the stringer and frames groups appears (Tab. 2a). Some considerations about this analysis follow. The optimal result is achieved with not violated constraints, so the new configuration is feasible in relation to the load cases and the requirement. The OPT.TOOL ends generating a optimal FEM with $0.74 \%$ mass increased. The last consideration is about the processing time: because of the high complexity of the solution and the type of optimization task activated (in particular on the panel stiffeners), the analysis lasts almost five hours. This processing time doesn't last so long as the MSC.Nastran run. In order to validate the OPT.TOOL procedure a comparison with MSC.Nastran's optimization solver is performed. The comparison between the MSC.Nastran and OPT.TOOL solution on the same FE model is based. As the finite element software can not support a sizing optimization of the distance between the panel stiffeners, the theory of equivalent stiffness is used to define the properties of the panels in the model and the panel stiffeners optimization is omitted. In comparison with to the previous example a different definition of the requirements are report: (1) all the load cases are activated; (2) specific range defined on the cross section of stringers and frames and panel thickness (respectively $64 \mathrm{~mm}^{2} \leq A_{i}\left(d_{i}\right) \leq 2500 \mathrm{~mm}^{2}$ and $\left.8 \mathrm{~mm} \leq t_{p} \leq 50 \mathrm{~mm}\right) ;$ (3) ultimate yield stress imposed to $270 \mathrm{MPa}$; (4) structural and stability security factors fixed respectively to $s_{1}=s_{2}=1.0$ : (5) because of the absence of the stiffener panel the local stability constraint is not taken into account on these elements. The comparison results show (Fig. 7) a small difference (about $20 \mathrm{~kg}$ ) between the two global weights (Tab. 2b), with a long processing time for the FE software. This is why the MSC.Nastran time processing will be longer if the time for writing the execution (Sol 200) will be considered; OPT.TOOL doesn't need this kind of user's attention because of its FE interfacing algorithm. A different mass distribution between the groups is reached because of the stability conditions that increase the panels weights giving out the remaining groups mass. 


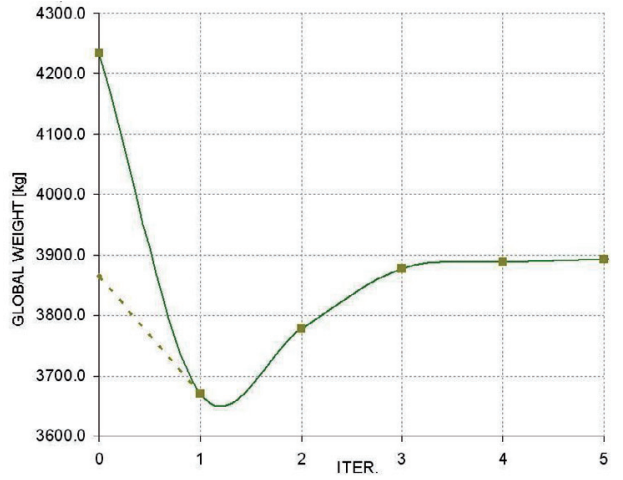

(a)

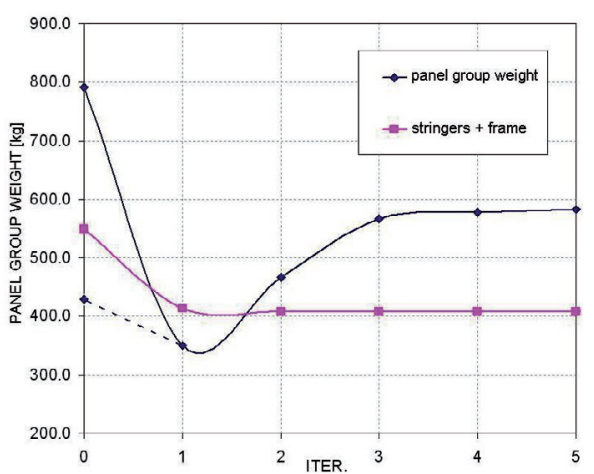

(b)

Fig. 8. OPT.TOOL space module analysis. Global (a) and local group weights (b) for the initial FE (dotted line) and the actual (continuous line) models.

Table 2. OPT.TOOL analysis (a) vs. MSC.Nastran comparison (b) results on a space structure.

\begin{tabular}{lcccc}
\hline & Start & OPT.TOOL(a) & OPT.TOOL(b) & MSC.Nastran(b) \\
\hline global weight [kg] & 3864.99 & 3891.82 & 3819.36 & 3798.69 \\
panels [kg] & 422.28 & 581.59 & 584.85 & 328.27 \\
str., fra. [kg] & 548,64 & 416.16 & 340.08 & 576,38 \\
time & - & $4^{h} 48^{m}$ & $2^{h} 21^{m}$ & $6^{h} 05^{m}$ \\
\hline
\end{tabular}

\section{Conclusions}

The work presents a multilevel optimization method for the preliminary design based on the $\mathrm{CO}$ architecture. It has been showed how the OPT.TOOL procedure can be a good approach for the sizing optimization of realistic structures defined by a FE model and considering some structural and stability local effects. Some features have been presented in two examples on aeronautical and aerospace structures, where: (1) a good comparison with a commercial optimization solver has been demostrated (with better results, in a shorter processing time); (2) new structural local configurations (wing example) and lighter model (aerospace example) have been founded respect to the initial conditions; (3) the process is completely automatic and no FE modifications are requested; (4) the OPT.TOOL local results are entirely uploaded in a FE model and available for further analyses.

\section{References}

1. R. Braun, R.W. Powell, R.A. Lepsch, I.M. Kroo, in Comparison of two multidisciplinary optimization strategies for launch-vehicle design, Journal Of Space And Rocket, Vol. 52, Nov. 2, May (2006)

2. O. Kalden, U.M. Schottle, in A software tool for analysis of future launch vehicle concepts, international Astronautical Congress Paper, IAC -03-V.5.08

3. N. Durante, A. Dufour, V. Pain, in Multidisciplinary analysis and optimization approach for the design of expendable launchers, 10th AIAA/ISSMO Multidisciplinary
Analysis and Optimization Conference, AIAA 2004-4441, 30 August-1 September, 2004

4. R. Braun, A. Moore, I. Kroo, in Use of the collaborative optimization architecture for launch vehicle design, AIAA Paper 96-4018, 1996

5. G.N. Vanderplaats, in An efficient feasible direction algorithm for design synthesis, AIAA Journal, Vol. 22, No. 11 (1984)

6. L. Guadagni, in Development of multilevel procedures for the optimal design of aerospace vehicles, Ph.D. Thesis, Politecnico di Torino, 2005

7. N.M. Alexandrov, R. M. Lewis, in Analytical and computational aspects of collaborative optimization, NASA Paper TM-2000-210104, 2000

8. N.M. Alexandrov, R.M. Lewis, In Analytical and computational properties of distributed approaches to $M D O$, 8th AIAA/USAF/NASA/ISSMO Symposium Of Multidisciplinary Analysis \& Optimization, September 2000

9. P. Piperni, M. Abdo, F. Katyeke, in The building blocks of a multi-disciplinary wing design method, General Meeting and Conference (Toronto), April 26 and 27, 2005

10. S. Craig, P.E. Collier, in Next generation structural optimization today, Colier Research \& Development Corporation, September 1997

11. N.F. Brown, J.R. Olds, in Evaluation of multidisciplinary analysis tecniques applied to a reusable launch vehicle, Journal Of Space And Rocket, Vol. 43, No. 6, (2006)

12. J. Wallace, J.R. Olds, A.C. Charania, G. Woodcock, in A studi of arts: a dual-fuel reusable launch vehicle with launch assist, 39th AIAA/ASME/SAE/ASEE Joint Propulsio Conference And Exibit, July 2003 